\title{
Artificial Neural Networks for the Prediction of Thermo Physical Properties of Liquid Mixtures
}

\author{
R. Baskaran \\ Department of Chemical Engineering \\ St.Joseph's college of Engineering \\ Chennai-119, India \\ Tel: 91-99-4128-5866Ｅ-mail: rbaskaran2000@yahoo.com \\ S. Arunachalam \& K. Manjunath \\ Department of Electrical and Electronics Engineering \\ St.Joseph's college of Engineering \\ Chennai-119, India \\ T.R Kubendran \\ Department of Chemical Engineering \\ Alagappa College of Technology \\ Anna University \\ Chennai-600025, India \\ E-mail: trkubendran@yahoo.co.in
}

\begin{abstract}
A predictive method based on Artificial networks has been developed for the thermophysical properties of binary liquid mixtures at $(303.15,313.15$ and 323.15) K. In method 1, a committee ANN was trained using 5 physical properties combined with absolute temperature as its input to predict thermo physical properties of liquid mixtures. Using these data, predicted values were determined for intermediate mole fraction of different systems without conducting experiments. In method 2, a committee ANN was trained using mole fraction and molecular weight as its input to predict the thermo physical properties of liquid mixtures. The five physical properties of five binary mixtures were taken for this study along with their molecular weights. ANN with back-propagation algorithm is proposed, for Multi-pass Turning Operation and developed in MATLAB. Compared to other prediction techniques, the proposed ANN approach is highly accurate and error is $<1 \%$.
\end{abstract}

Keywords: Artificial neural network, Neurons (nodes), Perceptron, Network training, Para anisaldehyde

\section{Introduction}

In many real world applications, we want our computer to perform complex recognition problems. Since our conventional computers are obviously not suited to this type of field, we therefore borrow features from the physiology has come to be known as Artificial Neural Systems (ANS) Technology or Neural Networks. Artificial neural network is a branch of artificial intelligence (AI) that attempts to achieve human brain like capability. Traditional approaches of solving chemical engineering problems frequently have their limitations, as for example in the modeling of highly complex and nonlinear systems. Artificial neural networks (ANN) have proved to be able to solve complex tasks in a number of practical applications. The utility of artificial neural network models lies in the fact that they can be used to infer a function from observations. This is particularly useful in applications where the complexity of the data or task makes the design of such a function by hand impractical. Because ANN are nets of basis functions, they can provide good empirical models of complex nonlinear processes useful for a wide variety of purposes. The applications of ANN include detection of medical phenomena, stock market prediction, credit assignment, monitoring the condition of 
machinery and engine management. There are only few reports of using ANN in the prediction of physiochemical properties, these reports have generally been restricted to equilibrium rather than transport properties (John.et.al 1999). It is believed that so far there has been no attempt to truly predict the properties of liquid mixtures across their wide temperature range using ANNs. This paper presents the findings of a programme of work devoted to the application of ANNs to thermo physical properties of binary mixtures. The thermo physical properties of binary mixtures are found by experimental work done by us. A predictive method based on Artificial Neural Networks (ANN) has been developed for ultrasonic velocity, density, kinematic viscosity, surface tension and refractive index for para anisaldehyde liquid mixtures over a wide range of mole fraction and temperatures. The study of various properties like viscosity, refractive index, density, surface tension and ultrasonic velocity for different systems are very important to understand the molecular interactions and purity of compounds. Using these experimental data, predicted data for intermediate mole fraction of different systems were generated, without conducting experiments.

\section{Working procedure}

A Neural Network is an interconnected assembly of simple processing elements, units or nodes, whose functionality is loosely based on the animal neuron. The processing ability of the network is stored in the inter-unit connection strengths, or weights, obtained by a process of adaptation to, or learning from, a set of training patterns. It has been shown that non linear feed forward neural networks are capable of universal functional approximation and that a single hidden layer is sufficient to uniformly approximate any continuous function Hornic.et.al.(1989). The neurons in a single hidden layer tend to interact globally but in complex functions this interaction makes it difficult to improve the approximation Heykin (1994), Maren .et.al (1990). The brain is principally composed of a very large number (circa 10,000,000,000) of neurons, massively interconnected (with an average of several thousand interconnects per neuron, although this varies enormously).

\subsection{Artificial neurons}

To capture the essence of biological neural systems, an artificial neuron is defined as follows:

- It receives a number of inputs (either from original data, or from the output of other neurons in the neural network). Each input comes via a connection that has a strength (or weight); these weights correspond to synaptic efficacy in a biological neuron. Each neuron also has a single threshold value. The weighted sum of the inputs is formed, and the threshold subtracted, to compose the activation of the neuron

- The activation signal is passed through an activation function (also known as a transfer function) to produce the output of the neuron.

In Figure 2.1, various inputs to the network are represented by the mathematical symbol, $\mathrm{x}(\mathrm{n})$. Each of these inputs is multiplied by a connection weight. These weights are represented by $\mathrm{w}(\mathrm{n})$. In the simplest case, these products are simply summed, fed through a transfer function to generate a result, and then output. This process lends itself to physical implementation on a large scale in a small package. The commonest type of artificial neural network consists of three groups or layers of units: input, hidden, and output. The layer of input neurons receives the data either from input files or directly from electronic sensors in real-time applications. The output layer sends information directly to the outside world, to a secondary computer process, or to other devices such as a mechanical control system Necat(2006). Between these two layers can be many hidden layers. These internal layers contain many of the neurons in various interconnected structures. The inputs and outputs of each of these hidden neurons simply go to other neurons.

\subsubsection{Prediction Of Thermo physical Properties}

Step 1: Collect data:

Things to be kept in mind while choosing the input data .

- The variables that are influential must be chosen

- Numeric and nominal variables can be handled. Convert other variables to one of these forms, or discard.

- Hundreds or thousands of cases are required; the more variables, the more cases.

Step 2: Define a network structure

An appropriate network topology is selected. Here for prediction problem, the neural network developed is fully connected feed forward multilayer perceptron. For this problem, the input variables are Temperature and mole fraction. So, the number of Input nodes is 2 . Here, we have to predict the density, kinematic viscosity, ultrasonic velocity, surface tension and refractive index of the systems. Hence, the number of output nodes is 5. After a number of experiments, the transfer function of the hidden layer and the number of epochs are set, the details of which are given below. For example consider a feed forward net with 30 hidden nodes in a single layer.

Step 3: Random initialization of weights and biases for the defined network 
Step 4: Back propagation learning Mandic (2001), Reilly (1982) algorithm is selected for training the network

Step 5: Training parameters are set

Number of iterations: 600

Performance goal: 0

Step 6: Start training

During training, the input and output data are presented to the network.

Step 7: Termination

The error for the network is calculated. The error calculation and weight updating process continues until the neural network reaches the performance goal or number of iteration.

\section{Results and Discussion}

Experimental thermo physical property values were extracted from the data base for all the mixtures studied. The list of binary liquid mixtures studied was displayed in Table 1. Input and output variables of method-1 and method -2 are listed in Table 2. The thermo physical properties were density, viscosity, refractive index, surface tension and ultrasonic velocity. The input to the ANN consisted of five thermo physical properties of five binary mixtures at $(303.15,313.15$ and 323.15) $\mathrm{K}$ for method - 1. The out put from the ANN consisted of five predicted thermo physical properties of five binary mixtures. In method -2 the input consisted of mole fraction, molecular weight and out put was thermo physical properties of five binary liquid mixtures. A BPN simulator is designed and the input data were fed in. BPN simulator is trained several times using selected data from the collected data, which consists of normal as well as abnormal data. During training, the simulator is presented with both input and output pairs and the error is generated which is the difference between actual and desired output. The error is minimized using the steepest descent technique. When the error obtained is of acceptable value, then the simulator is said to be trained. Then, the data for prediction is presented to the neural network after training. The mean square error is calculated using BP algorithm and the learning curve is plotted between the mean square error and the number of generations (iterations) i.e. epochs. Performance and error graph of method -1 and 2 of the trained committee ANN for the binary systems are presented in Figure 3.1- 3.10. While examining the results of these various neural networks, they were found to be in agreement with the desired results and within permissible error range. The algorithms traingdm or traingd are not producing satisfactory results for the 2 X 20 X 10 X 5 neural network for the maximum epochs of 800 and for a performance goal of 0 . The trained algorithm with a single hidden layer with 30 nodes i.e 2 X 30 X 5 neural network. (Figure2.1.1) is producing the desired result for the prediction of these properties. The predicted values of other new mole fractions of the mixtures were taken and verified experimentally in Table 3 . The percentage of error is $<1$.

\section{Conclusion}

Due to high speed of processing, low consumption of memory, great robustness, possibility of self learning and simple incorporation into chips the approach ensures prediction condition in real time. It provides robust representation clue to the fault-tolerant nature of neural networks. When comparing methods 1 and 2, the first method holds good which is indicated by low error. Our future proposal is Extending the proposed idea of ANN prediction to systems at extremely high or low temperatures where conducting of experiments are difficult.

\section{References}

John, H., Sotos, C.G., John, H.R. (1999). Artificial neural networks for the prediction of liquid viscosity, density, heat of vaporization, boiling point and pitzers acentric factor. Phys.Chem.Chem.Phys. 1, 4075-4081.

Hornik, K., Stinchcombe, M., White, H.(1989). Multilayer feed forward neural networks are Universal approximators. Neuron Networks, 2, 359-366.

Heykin,S.( 1994 ). Neural network a comprehensive foundation, New York, ch.6.12.

Maren, A., Harston,C., Pap,R. (1990).Handbook of neural computing applications, New York, 238.

Necat Altinkok. (2006). Use of Artificial Neural Network for Prediction of Mechanical Properties of $-\mathrm{Al}_{2} \mathrm{O}_{3}$ Particulate-reinforced Al-Si10Mg Alloy Composites Prepared by using Stir Casting Process. Journal of Composite Materials, 40, 9, 779-796.

Mandic, D., Chambers, J. (2001). Recurrent Neural Networks for Prediction: Architectures, Learning algorithms and Stability. Wiley.

Reilly, D.L., Cooper, L.N., Elbaum, C. (1982). A Neural Model for Category Learning. Biological Cybernetics 45, $35-41$. 
Table 1. Details of the systems studied

\begin{tabular}{|c|c|c|c|c|}
\hline Sl.no & systems & Temperatures & $\begin{array}{l}\text { Net work } \\
\text { topology }\end{array}$ & $\begin{array}{l}\text { Training } \\
\text { algorithm }\end{array}$ \\
\hline 1 & Anisaldehyde + chlorobenzene & \multirow{5}{*}{$\begin{array}{l}303.15,313.15 \\
\text { and } 323.15 \mathrm{~K}\end{array}$} & \multirow{5}{*}{$\begin{array}{l}\text { Feed forward } \\
\text { multilayer } \\
\text { perceptron }\end{array}$} & \multirow{5}{*}{$\begin{array}{l}\text { Back propagation } \\
\text { learning algorithm }\end{array}$} \\
\hline 2 & Anisaldehyde + bromobenzene & & & \\
\hline 3 & Anisaldehyde + nitrobenzene & & & \\
\hline 4 & Anisaldehyde + ethyl benzene & & & \\
\hline 5 & Anisaldehyde + benzene & & & \\
\hline
\end{tabular}

Table 2. Input and output variables of method - 1 and method - 2

\begin{tabular}{|l|l|l|l|}
\hline \multicolumn{2}{|l|}{} & \multicolumn{2}{|}{ Method -2 } \\
\hline Input & Output & Input & Output \\
\hline $\begin{array}{l}\text { Mole fraction } \\
\text { Experimental } \\
\text { five Thermo } \\
\text { physical } \\
\text { properties }\end{array}$ & $\begin{array}{l}\text { Predicted five } \\
\text { thermo physical } \\
\text { properties }\end{array}$ & Mole fraction & $\begin{array}{l}\text { Polecular weight } \\
\text { physical properties }\end{array}$ \\
\hline
\end{tabular}

Table 3. Random experimental verification of ANN predicted thermo physical properties (not included in the training data) of mixtures with percent standard deviation.

\begin{tabular}{|c|c|c|c|c|c|c|c|c|c|c|c|c|}
\hline Systems & $\mathrm{x}_{1}$ & $\mathrm{~T} / \mathrm{K}$ & $\begin{array}{l}\text { P } \\
\text { (pre) }\end{array}$ & S \% & $\begin{array}{l}\eta \\
\text { (pre) }\end{array}$ & $\mathrm{S} \%$ & $\begin{array}{l}\mathrm{n}_{\mathrm{D}} \\
\text { (pre) }\end{array}$ & $\begin{array}{l}\mathrm{S} \\
\%\end{array}$ & $\begin{array}{l}\sigma \\
\text { (pre) }\end{array}$ & S \% & $\begin{array}{l}\mathrm{u} \\
\text { (pre) }\end{array}$ & $\begin{array}{l}S \\
\%\end{array}$ \\
\hline \multirow{3}{*}{$\begin{array}{l}\text { Anisaldehyde } \\
\text { and benzene }\end{array}$} & 0.1500 & 303.15 & 0.890 & 0.28 & 0.825 & 0.48 & 1.509 & 0.37 & 37.21 & 1.72 & 621 & 0.20 \\
\hline & 0.5000 & 313.15 & 1.000 & 0.98 & 1.214 & 0.42 & 1.524 & 1.40 & 64.21 & 0.58 & 998 & 0.47 \\
\hline & 0.9000 & 323.15 & 1.100 & 0.99 & 2.610 & 0.91 & 1.544 & 0.81 & 77.61 & 0.85 & 1600 & 0.21 \\
\hline \multirow{3}{*}{$\begin{array}{l}\text { Anisaldehyde } \\
\text { and chloro } \\
\text { benzene }\end{array}$} & 0.1500 & 303.15 & 1.108 & 0.85 & 0.945 & 0.95 & 1.558 & 0.89 & 47.91 & 0.65 & 1361 & 1.14 \\
\hline & 0.5000 & 313.15 & 1.100 & 0.98 & 1.252 & 0.59 & 1.550 & 2.60 & 68.71 & 0.74 & 1121 & 1.27 \\
\hline & 0.9000 & 323.15 & 1.110 & 1.14 & 1.999 & 0.98 & 1.546 & 1.96 & 88.21 & 0.12 & 1405 & 1.11 \\
\hline \multirow{3}{*}{$\begin{array}{l}\text { Anisaldehyde } \\
\text { and } \\
\text { bromobenzene }\end{array}$} & 0.1500 & 303.15 & 1.421 & 2.18 & 0.988 & 2.48 & 1.558 & 3.04 & 44.21 & 2.04 & 1260 & 2.00 \\
\hline & 0.5000 & 313.15 & 1.300 & 2.26 & 1.344 & 2.67 & 1.550 & 2.11 & 64.21 & 2.12 & 1364 & 2.54 \\
\hline & 0.9000 & 323.15 & 1.155 & 2.18 & 2.125 & 2.98 & 1.547 & 2.25 & 88.21 & 2.79 & 1521 & 2.91 \\
\hline \multirow{3}{*}{$\begin{array}{l}\text { Anisaldehyde } \\
\text { and } \\
\text { nitrobenzene }\end{array}$} & 0.1500 & 303.15 & 1.190 & 0.14 & 2.014 & 0.35 & 1.553 & 0.11 & 44.21 & 0.24 & 1461 & 1.02 \\
\hline & 0.5000 & 313.15 & 1.154 & 0.62 & 2.125 & 0.49 & 1.548 & 3.28 & 61.21 & 0.18 & 1468 & 1.05 \\
\hline & 0.9000 & 323.15 & 1.116 & 0.19 & 2.366 & 0.42 & 1.546 & 3.15 & 80.18 & 0.19 & 1547 & 1.00 \\
\hline \multirow{3}{*}{$\begin{array}{l}\text { Anisaldehyde } \\
\text { and } \\
\text { ethylbenzene }\end{array}$} & 0.1500 & 303.15 & 0.922 & 0.85 & 1.320 & 0.11 & 1.510 & 1.11 & 42.21 & 0.00 & 1402 & 1.06 \\
\hline & 0.5000 & 313.15 & 1.001 & 0.13 & 1.910 & 0.41 & 1.520 & 1.15 & 70.12 & 0.00 & 1457 & 1.07 \\
\hline & 0.9000 & 323.15 & 1.077 & 0.61 & 2.301 & 0.20 & 1.540 & 0.01 & 92.95 & 0.04 & 1532 & 1.08 \\
\hline
\end{tabular}




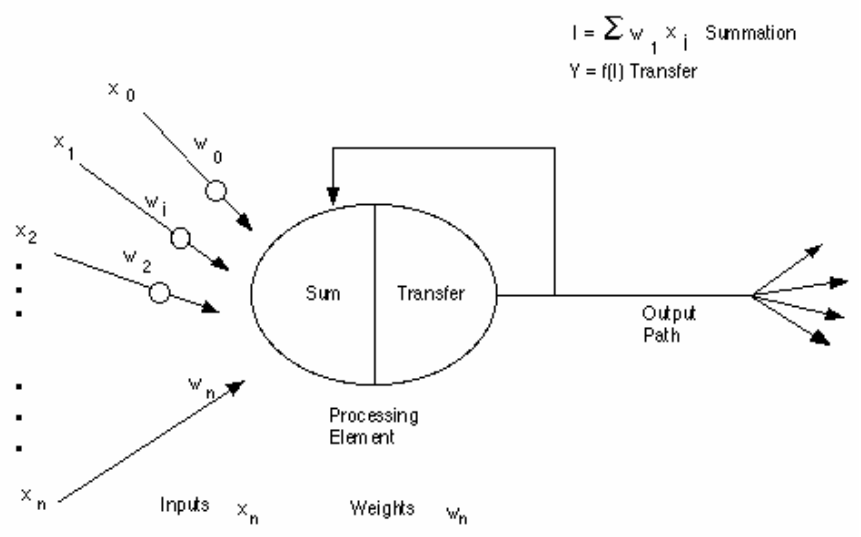

Figure 2.1 A Basic Artificial Neuron.

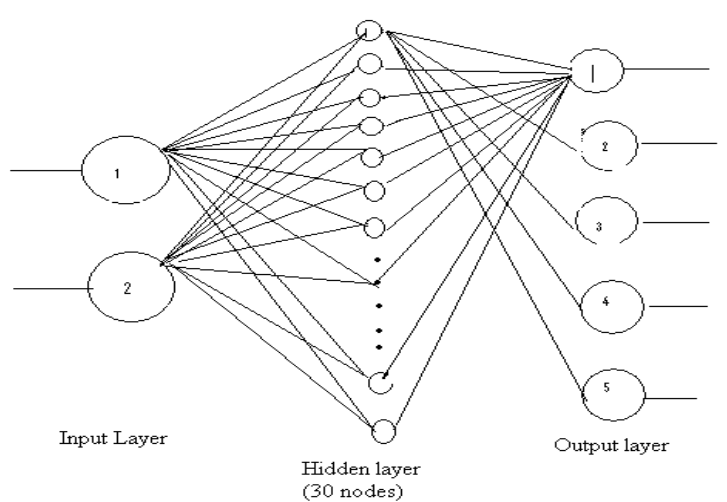

Figure 2.1.1 Feed forward net work

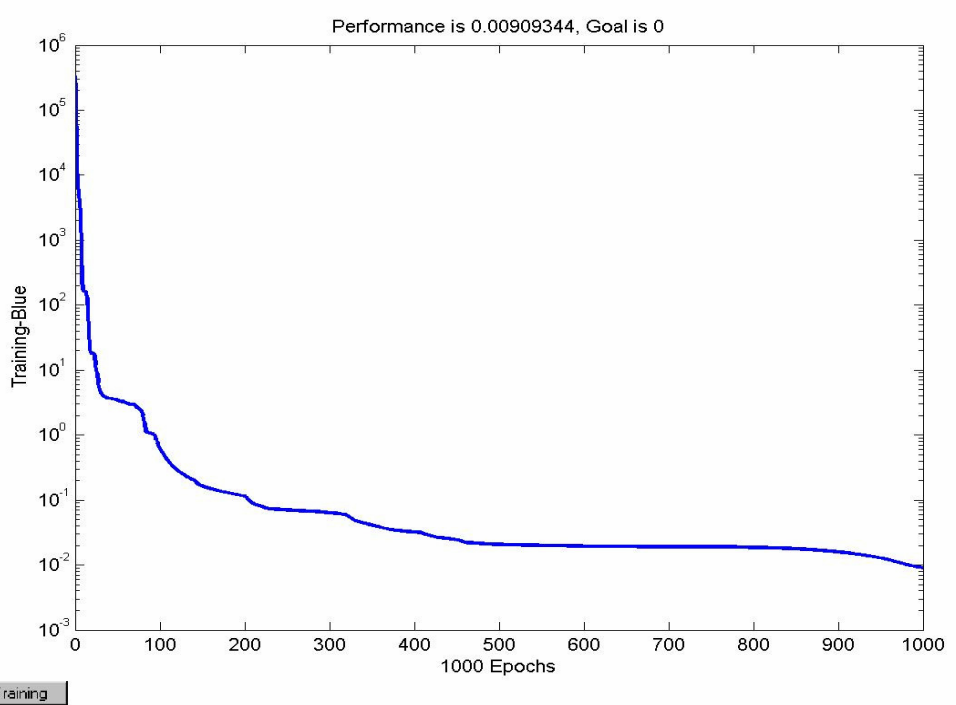

Figure 3.1 Plot of error and number of iterations - Error performance for anisaldehyde-chlorobenzene mixture 


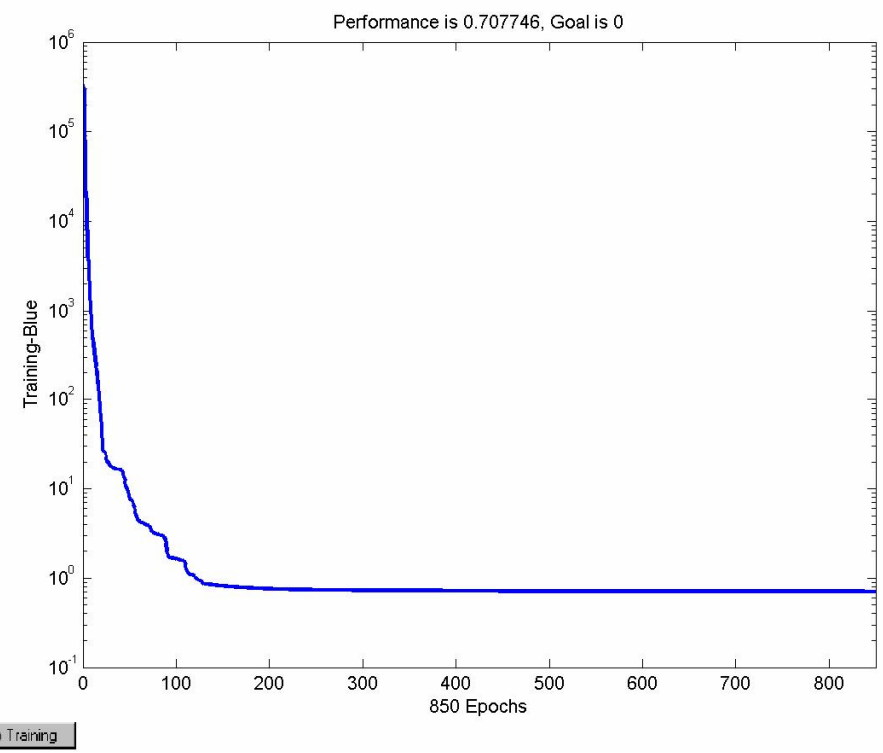

Figure 3.2 Plot of error and number of iterations - Error performance for anisaldehyde-chlorobenzene system with molecular weight.

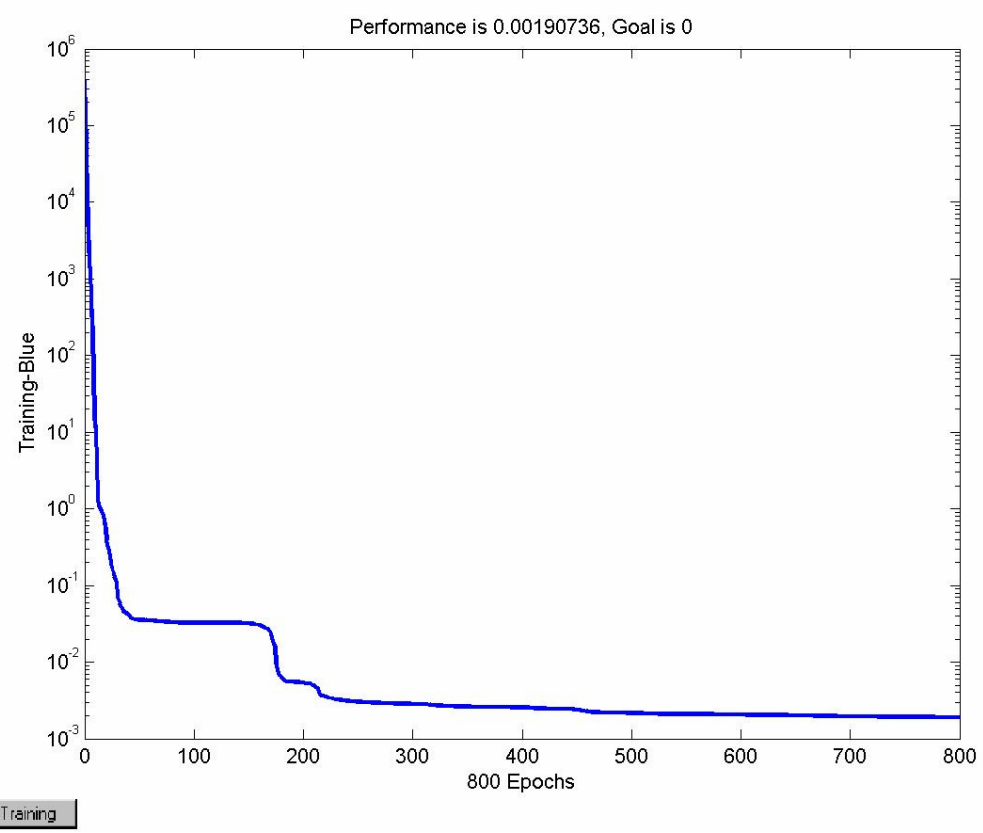

Figure 3.3 Plot of error and number of iterations - Error performance for anisaldehyde-bromobenzene system 


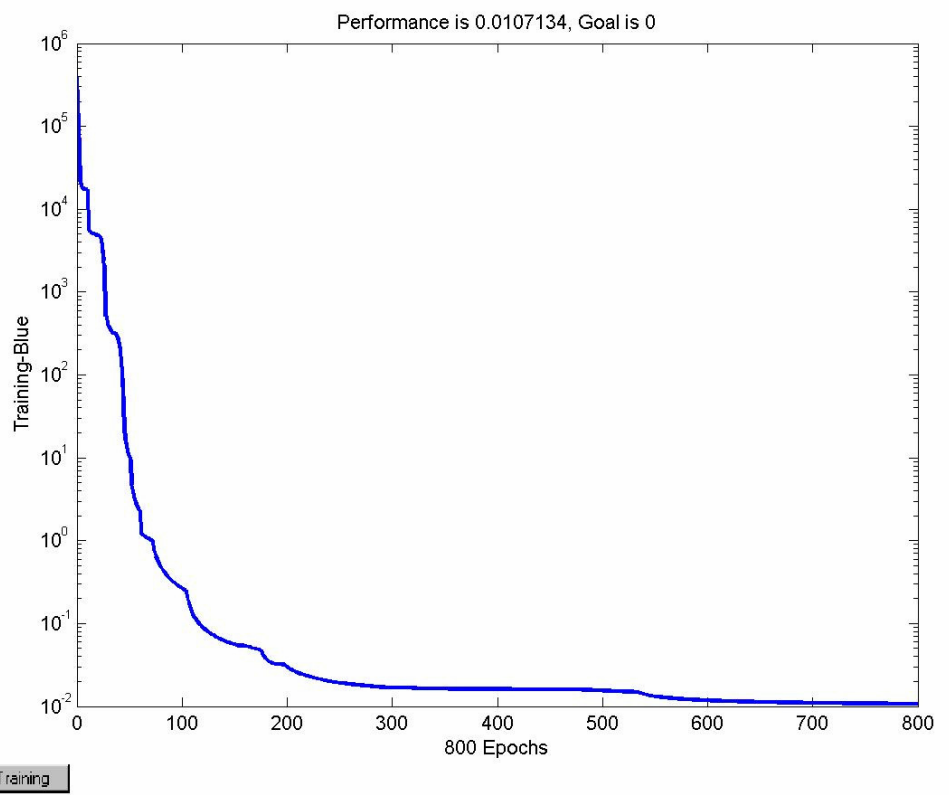

Figure 3.4 Plot ot error and number of 1terations - Error pertormance tor anısaldehyde-bromobenzene system with molecular weight

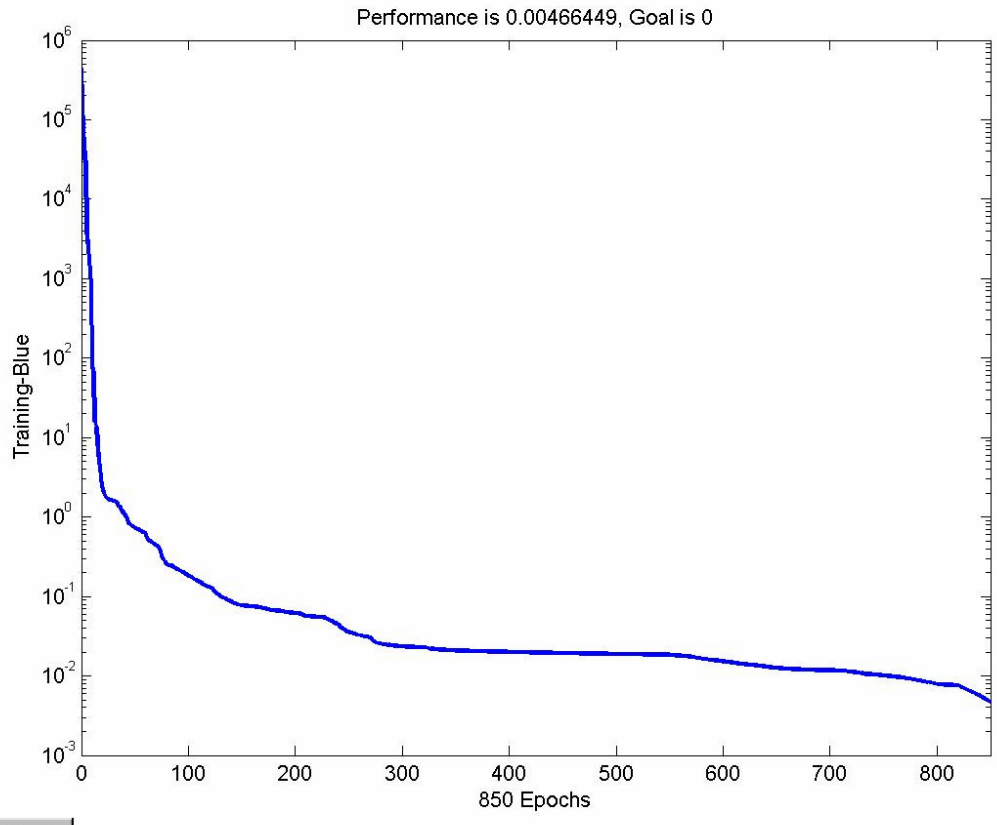

Stop Trairing

Figure 3.5 Plot of error and number of iterations - Error performance for anisaldehyde-nitrobenzene system 


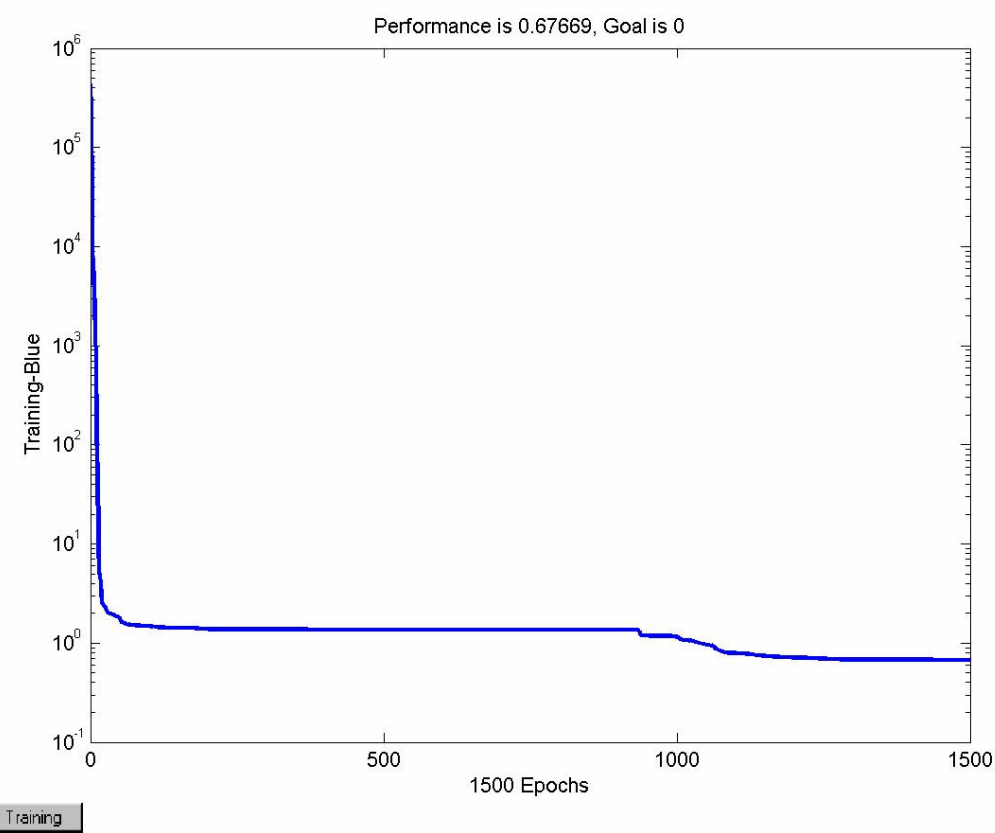

Figure 3.6 Plot of error and number of iterations - Error performance for anisaldehyde-nitrobenzene system with molecular weights

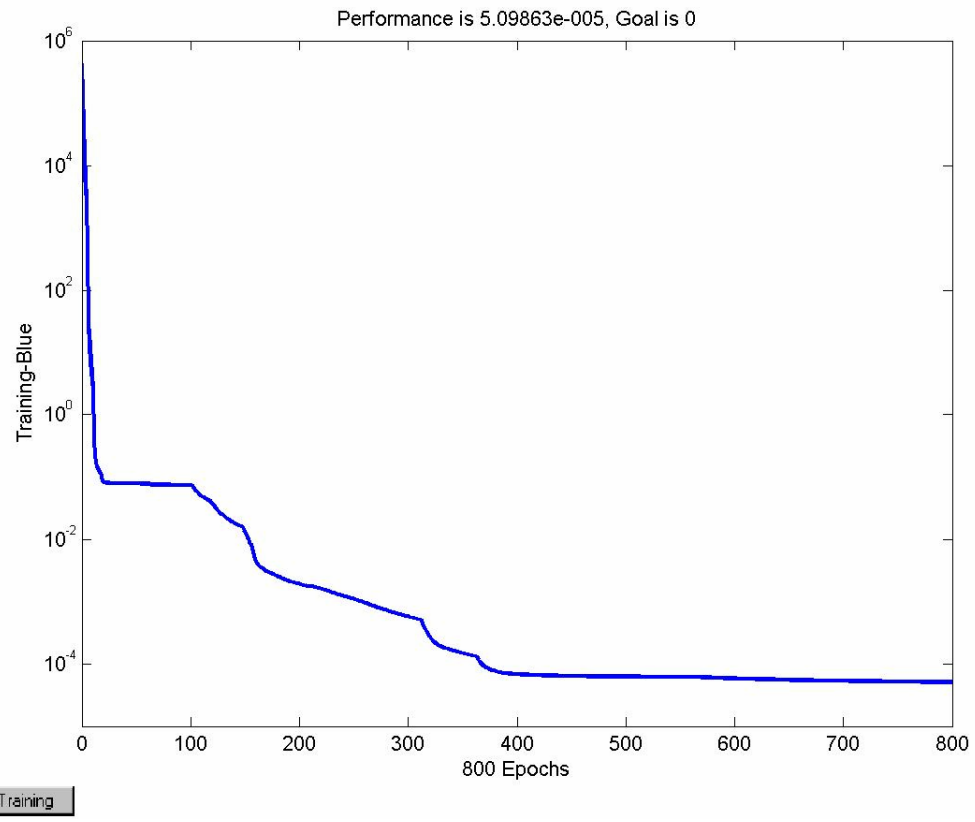

Figure 3.7 Plot of error and number of iterations - Error performance for anisaldehyde-ethylbenzene system 


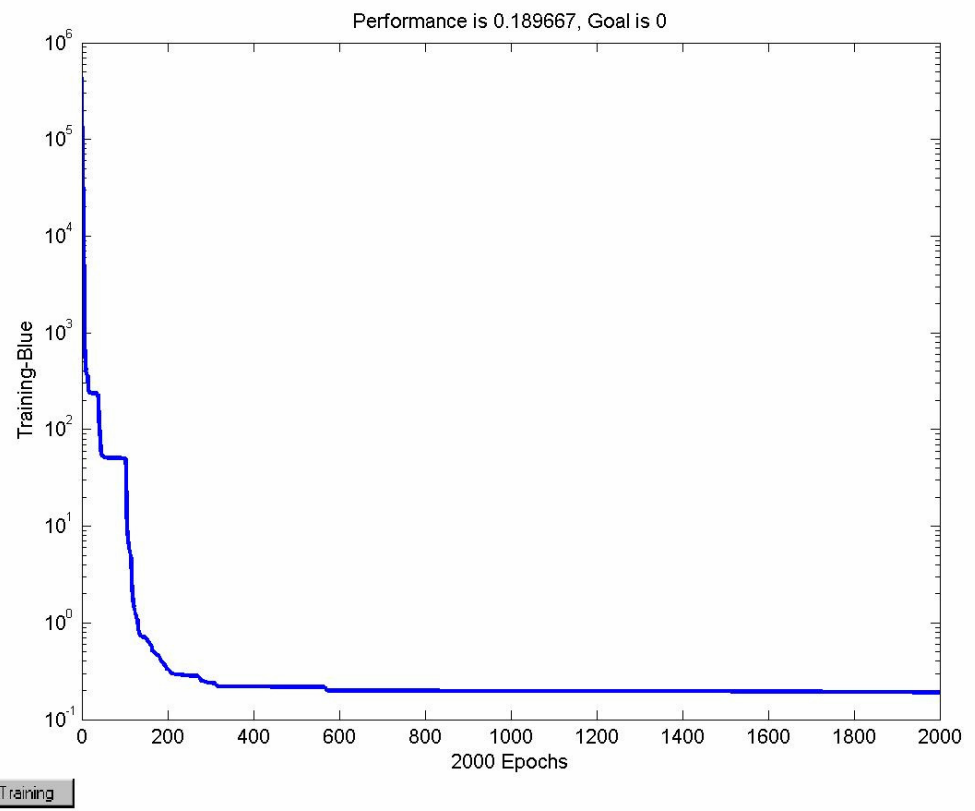

Figure 3.8 Plot of error and number of iterations - Error performance for anisaldehyde-ethylbenzene system with molecular weights

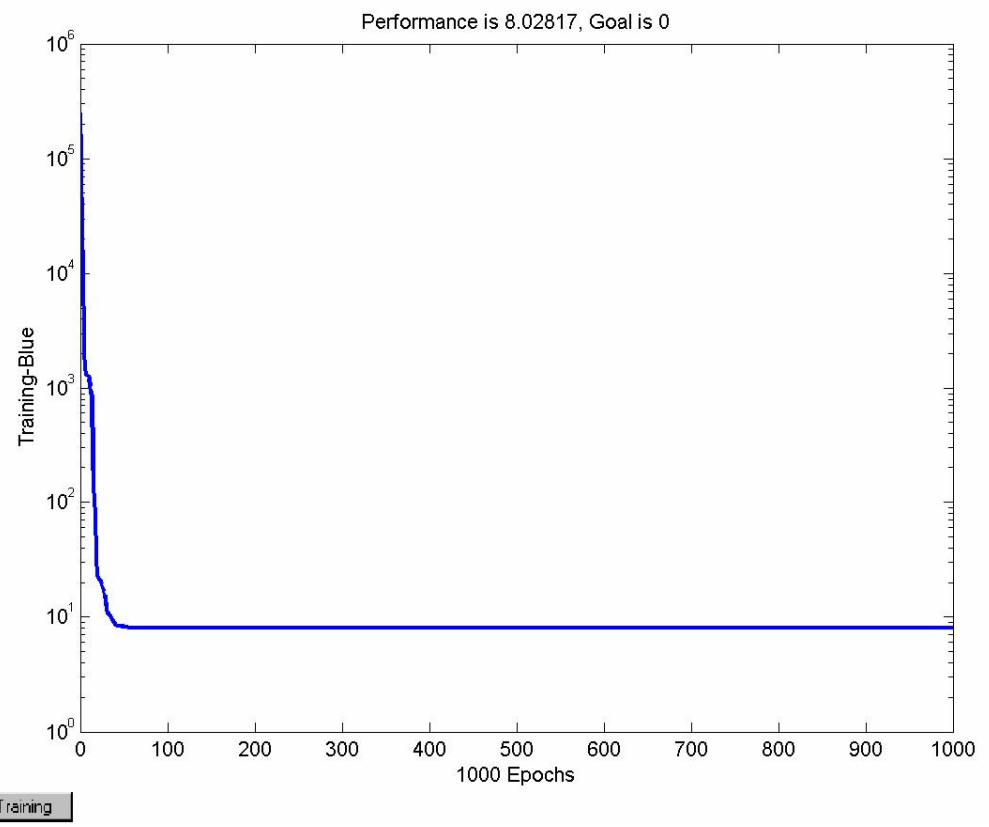

Figure 3.9 Plot of error and number of iterations - Error performance for anisaldehyde-benzene system 


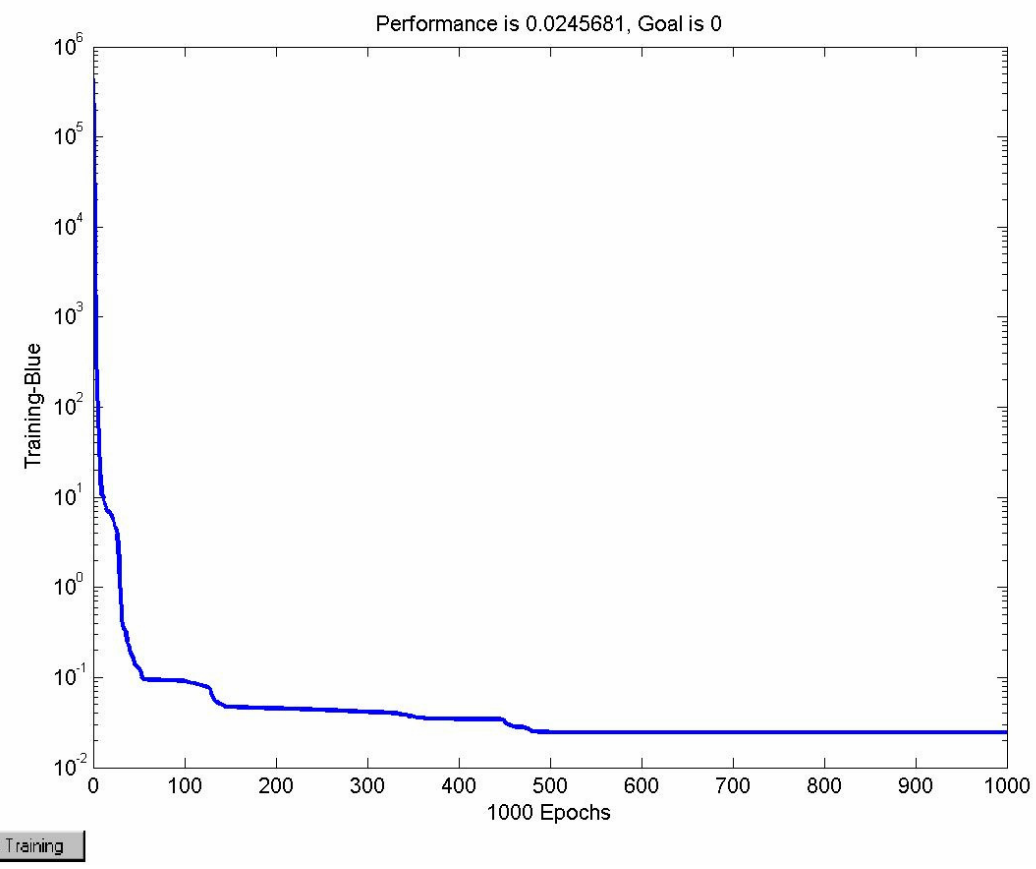

Figure 3.10 Plot of error and number of iterations - Error performance for anisaldehyde-benzene system with molecular weights 